\title{
Wellbore Cool Down During Hydraulic Fracturing Simplifies Using Dissolvable Materials
}

\author{
Michael Fripp*, Zachary Walton and Halliburton \\ Halliburton Energy Services, USA \\ *Corresponding author: Michael Fripp, Halliburton Energy Services, USA.
}

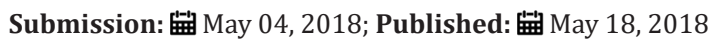

\begin{abstract}
Dissolvable materials are enabling a revolution in the completion and stimulation of wellbores. Effective design of dissolvable fracturing tools, however, needs to account for the substantial cooling effect that arises during pump down and stimulation. The degradation process, degradation time, and material properties change because of the wellbore cool down. A well bore cools when fluids are injected into the formation. Temperature profiles are measured with fibre-optic cable and are simulated with numerical models. Both measured and modelled results show that down hole temperatures can be cooled to near surface temperatures. This temperature data is combined with experimental dissolution behavior to show that different dissolution behaviour occurs during pump down, setting, wellbore stimulation, and wellbore temperature recovery.

The actual temperature that fracturing tools will experience during their useable life depends on wellbore cool down and can be much less than the static formation temperature. All dissolvable materials have dissolution rates and material properties that are temperature dependent. Some dissolvable plastics will not degrade at lower temperatures. Measured and modelled cool down from hydraulic fracturing are used to show the temperature profile during different operational stages. The greatest temperature cool down occurs while the dissolvable fracturing tool is isolating a zone during the fracture treatment. This temperature cool down also occurs during the only time of the operation in which the fracturing tools are required to maintain a significant differential pressure. The temperature cool-down effect becomes more significant as more zones are fractured and more fluid is injected into the formation. Dissolvable fracturing tools should be designed for the temperature experienced during wellbore cool down rather than the static formation temperature. Both field data and numerical modelling show that a dynamic temperature estimation is more accurate than static bottom hole temperature (BHT) at predicting the temperature experienced by dissolvable fracturing tools. The cool down of the wellbore is an important design parameter for the materials and construction of dissolvable tools.
\end{abstract}

\section{Introduction}

Dissolvable materials are enabling a revolution in terms of the completion and stimulation of wellbores. The advantage of dissolvable materials is particularly evident in their use for hydraulic fracturing where the frac plugs and the frac balls can be constructed entirely from dissolvable materials. Unconventional reservoirs, such as shale formations, typically become economical when the surface area of the reservoir contact is increased, and one of the most effective and economical methods of increasing the reservoir contact is through multistage hydraulic fracturing [1]. Frac plugs and balls are used to isolate previously fractured zones and to direct the flow to the next fracture stage. In the plug-andperf approach to hydraulic fracturing, the frac plug and guns are pumped into the wellbore to the location where zonal isolation is necessary [2]. After the plug is set, perforations are made in the casing above the frac plug using perforation guns and completion fluid and sand are pumped into the wellbore to stimulate the perforated zone while the frac plug isolates the zones below.
This method of plug-and-perf is repeated until the target number of pay zones has been completed. However, the frac plugs remain in the wellbore, which can create restrictions to production. Full production requires that the frac plug be removed. Traditionally, composite type frac plugs were frequently used because they could be easily milled out [2]. New dissolvable frac plugs reduce the likelihood of needing to mill out the plugs. These dissolvable plugs are constructed from dissolvable metal, plastic, and elastomer. They are designed to dissolve away in typical completion fluids once the fracturing operation is completed, thus eliminating the milling process [3]. If dissolvable type frac plugs were used during fracturing, then a lower cost wellbore cleanup can be used rather than the process used for a longer duration milling operation. Dissolvable fracturing tools allow for lower cost hydraulic fracturing with a quicker time to production and allows for the construction of extended reach laterals [4]. 
An often over looked aspect of pumping fluids into a well bore, such as during hydraulic fracturing, is that the injected fluids will significantly reduce the temperature of the well bore. The cooling effect of circulating mud during drilling has been measured and numerically modelled [5] and has implications for borehole stability [6]. During acid stimulation, distributed fiber-optic measurements have demonstrated the cooling effect of the injected fluid [7] and have used this distributed measurement to determine where the acid is entering the formation [8,9]. Similarly, distributed fibre optics has been used to describe the cooling effects during hydraulic fracturing $[10,11]$. Most of these temperature diagnostics have been used to determine how the formation is accepting the injected fluids [12]. During the fracturing operation, the wellbore cool down has implications on the design and expected performance operation of dissolvable tools. The temperature of the frac plugs and balls will be at an intermediate temperature between the surface and the formation temperatures. The dynamic temperature will strongly influence the rate at which the dissolvable materials will disappear and can have a strong influence on the strength of the materials.

The recent introduction of dissolvable frac plugs gives this technology a short history compared to the long historical success of the composite frac plugs. As a result, a fundamental question arises-what should be the design temperature for a dissolvable frac plug? How significant is the cooling effect of pumping the frac plug into the wellbore and how does the cooling effect of the stimulation changes the down hole temperature? Should pressure testing of fracturing tools occur at formation, room, or at intermediate temperatures? This paper provides a framework to evaluate the proper temperature for the design and testing of dissolvable fracturing tools.

\section{Temperature During Hydraulic Fracturing}

The wellbore is cooled significantly when the frac plugs are runin-hole (RIH) and during stimulation. During installation, the frac plug is typically pumped into the horizontal section with a surfacetemperature fluid that will reduce the temperature of the wellbore. During stimulation, hydraulic fracturing fluids are pumped at high rates and can significantly reduce wellbore temperature. The highest pressure differential on the frac plug occurs when the wellbore is being cooled by the frac fluids. After fracturing is completed, the wellbore eventually returns to formation temperature. The key question is how much cooling will occur during the different phases of the hydraulic fracturing operation.

Numerical modelling can be used to predict the temperature cool down. Coupled real-time stimulation temperature modelling shows that hydraulic fracturing will cool the casing to the fracture fluid temperature within a minute of the arrival of the fracture fluids at location [8]. The cooling is particularly rapid at the modelled 69$\mathrm{bbl} / \mathrm{min}$ pump rate. The coupled simulation also shows that the reservoir will return to formation temperature in 1 to 10 hours, depending on the size of the fracture. Additional thermal models indicate that the casing will be within $10^{\circ} \mathrm{F}$ of the fracture fluid temperature at much lower stimulation rates, for some simulation of a Bakken Formation down to 2-bbl/min pump rates [13].

A commercial thermal and flow simulator [14] was used to predicted the temperature and pressure profile for a hydraulic fracturing operation in a representative Middle Eastern formation (Figure 1). Table 1 note the operational steps used during the simulation of the hydraulic fracturing stimulation. The simulation shows that the wellbore temperature at the frac plug is cooled by the injected fluids during the pumping of the frac plug to location, Event 1, and during the treatment of the zone, Events 4 and 5. The models predict that the frac plug is not exposed to the formation well temperature when the maximum differential pressure is applied to the frac plug. The temperature at the frac plug, however, is higher than the modelled $80{ }^{\circ} \mathrm{F}$ temperature of the injected fluid because of the distance between the lowest perforation and the frac plug.

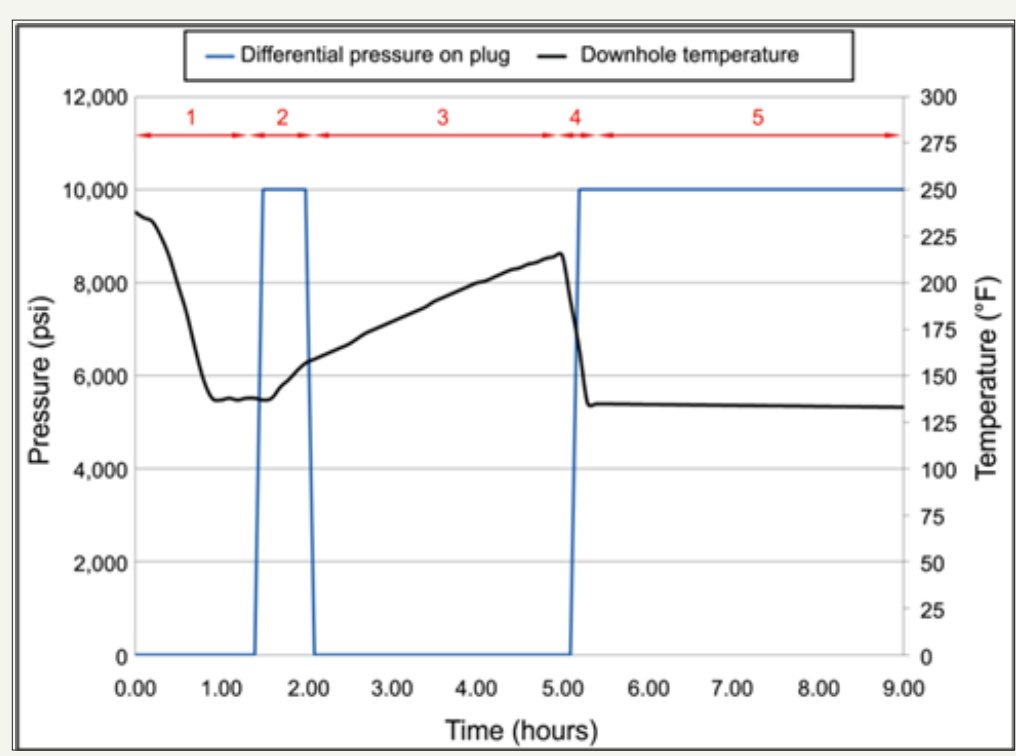

Figure 1: Simulated temperature and pressure response during hydraulic fracturing. 
Table 1: Events during a simulated hydraulic fracturing stimulation.

\begin{tabular}{|c|c|c|c|c|}
\hline Event & Event Description & Event Start & Event Duration & Total Duration \\
\hline 1 & Pump frac plug down to setting depth & $0: 00$ & $1: 30$ & $1: 30$ \\
\hline 2 & Set frac plug. Conduct initial pressure test & $1: 30$ & $0: 28$ & $1: 58$ \\
\hline 3 & Fire guns. Pull guns out of hole; rig up frac & $1: 58$ & $2: 38$ & $4: 36$ \\
\hline 4 & Begin zone treatment; pump frac ball & $4: 36$ & $0: 15$ & $4: 51$ \\
\hline 5 & Hydraulic fracturing & $4: 51$ & $3: 35$ & $8: 26$ \\
\hline
\end{tabular}

The thermal cool down during hydraulic fracturing can be measured directly with a fiber-optic cable. Distributed temperature sensing (DTS) examines the backscatter from light pulses in the fiber-optic cable to estimate the temperature along the fiber. Figure 2 presents the measured temperature profile during a hydraulic fracturing operation [10]. The fracturing fluids are exiting the casing through the perforations at 6,020 and 6,270ft. The wellbore at and below the packer is already warming following the conclusion from the previous stage of fracturing. The variability in the DTS measurements reflects variations in the thermal coupling between the fiber optic and the casing; better thermal coupling is obtained at the cable clamps. The frac plug will be deeper in the wellbore than the lowest perforation and, thus, will be warmer than the fluid temperature.

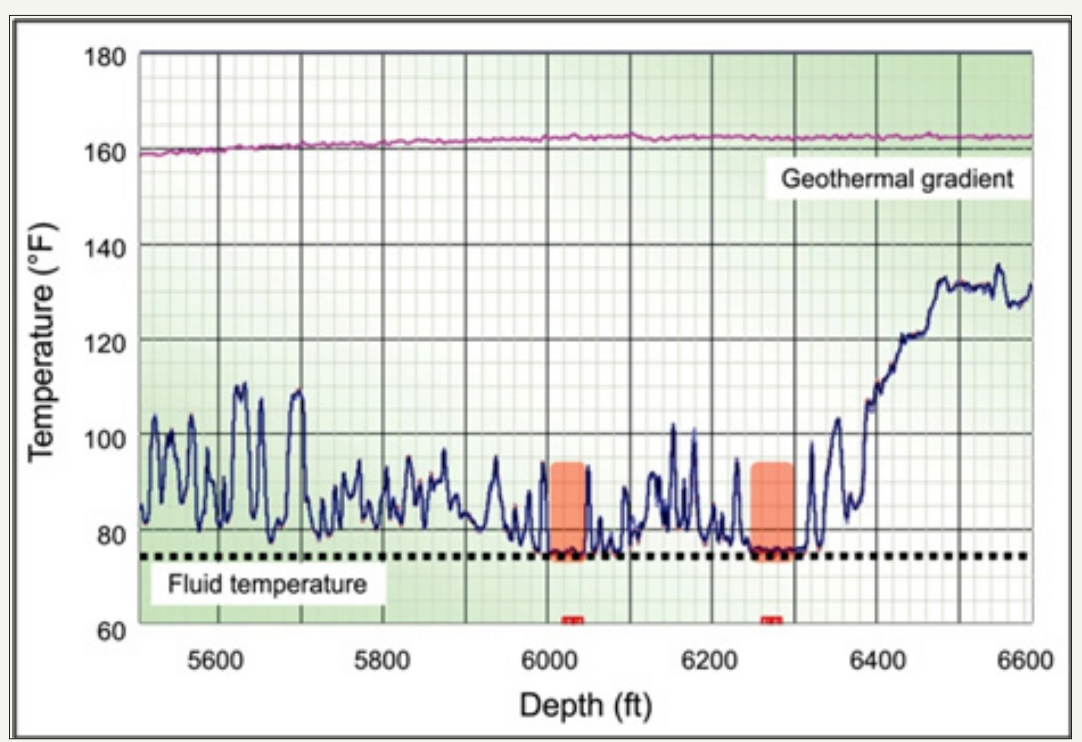

Figure 2: Events during a simulated hydraulic fracturing stimulation [10].

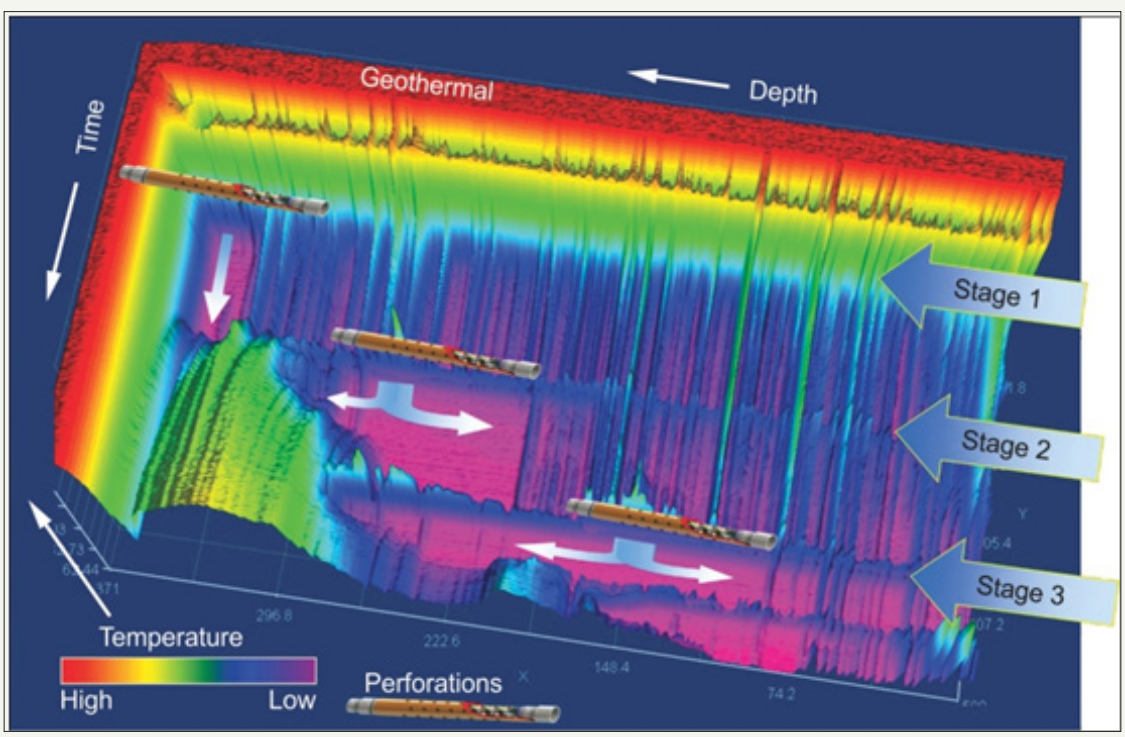

Figure 3: Measured temperature profile from three stages of hydraulic fracturing [10]. 
Figure 3 illustrates a three-dimensional color map of the measured down hole temperature showing three sequential stages of hydraulic fracturing. The wellbore is rapidly cooled from the static geothermal temperature by the injected fluids. At the perforations, the fiber-optic cable is measuring a temperature that matches the injected fluid temperature. After Stage 1 is completed, the first zone rapidly starts to return towards the geothermal gradient. Stage 3 show that there is fluid communication behind the casing which results in cooling deeper in the wellbore.

The numerical simulations and the measured fiber-optic data both show that the wellbore is rapidly and substantially cooled by the injected fracturing fluid. The temperature at the frac plug is substantially cooler than the static down hole temperature. The temperature at the perforations nearly matches the surface temperature of the injected fluid. However, the temperature at the frac plug is warmer than at the perforations and is warmer than the stimulation fluids. The stimulation fluids exit the casing at the perforations, which are a distance away from the frac plug. The distance between the frac plug and the deepest perforation allows for heat flux from the formation to increase the temperature around the frac plug.

\section{Dissolvable Materials}

A variety of dissolvable materials have been developed for hydraulic fracturing tools, including metals, elastomers, and plastics [15]. The strength and the dissolution rate of all of these materials depend on the actual temperature around the tool. Dissolvable metals generally degrade through an internal galvanic reaction in the metal. Dissolvable metals use variations in the local galvanic corrosion to achieve controlled dissolution. Increasing the galvanic coupling, such as through the addition of a cathodic dopant, will increase the rate that the metal degrades.

Dissolvable metals can be optimized for different temperature regimes by varying the concentration of the cathodic dopant. In general, higher concentrations of the cathodic dopant will lead to higher degradation rates and these high-dopant materials are best suited for low-temperature applications. For high-temperature applications, generally a lower concentration of the cathodic dopant is added to the metal. As presented in Figure 4, the galvanic corrosion occurs at the interface between the base metal and the cathodic sites and results in micro-galvanic corrosion of the base metal. The cathodic phases are minimally affected by the corrosion and will shed to create a fine powder of micron-scale dust. This fine powder will not interfere with flow back or with production. Increasing the concentration of the cathodic dopant will increase the number of cathodic sites and will increase the dissolution rate of the dissolvable metal.

The dissolution rate of the dissolvable metal will increase with increasing temperature (Figure 5). The base rate will vary with the amount of cathodic dopant, but the temperature sensitivity is consistent across different dopant concentrations. Knowledge of the actual temperature around the frac plug is important for knowing the time that the plug will dissolve.

The material strength of the dissolvable metals is also a function of temperature. Figure 6 presents the relative yield strength for two different formulations of dissolvable metal. The yield strength decreases at the temperature increases. Changing the concentration of the cathodic dopant will change the relative yield strength as well as changing the sensitivity of the yield strength with temperature. Dissolvable elastomers and plastics degrade through a hydrolytic cleaving of the polymer bonds. Hydrolytic cleaving reduces the strength of the polymers and can be monitored by noting the decrease in hardness of the polymer. Figure 7 presents the dissolution of a low-temperature elastomer, which shows strong temperature sensitivity to the rate of dissolution [16]. (a)

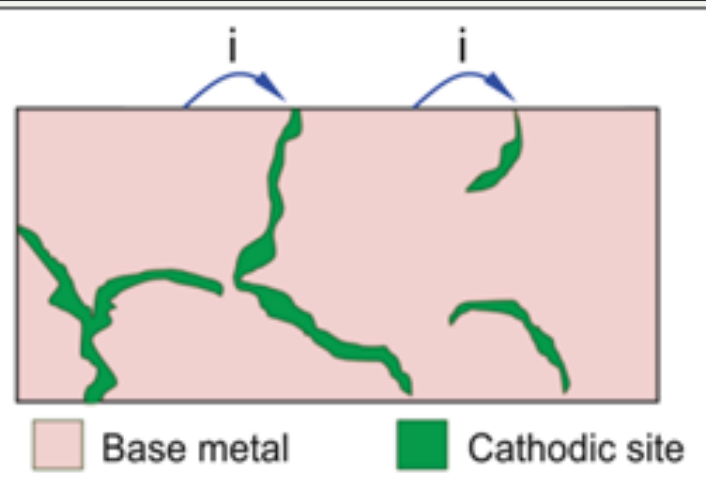

(b)

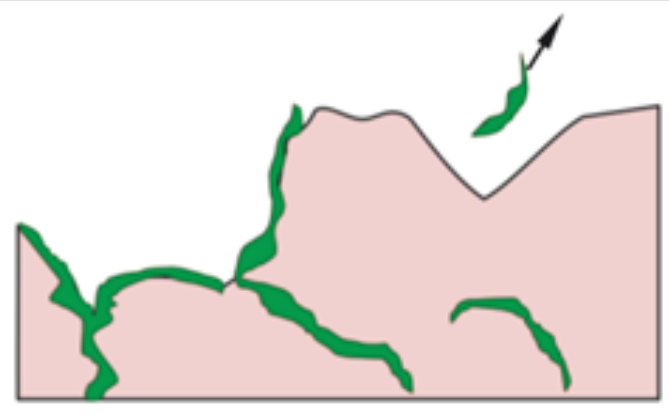

Figure 4: Schematic presentation of the degradation between the base metal and the cathodic phase;

(a) Micro-Galvanic corrosion of base metal; (b) intermediate corrosion shape.

Dissolvable polymers tend to follow an Arrhenius-type temperature dependence, which is expected based on the chemistry of their degradation drivers [17]. Dissolvable plastics tend to follow Arrhenius-type temperature dependence until the glass transition temperature is reached. For dissolvable plastics, such as those based on polyglycolic acid (PGA) chemistry, the degradation rate is greatly slowed once the material temperature is less than the glass transition temperature. At temperatures above the glass transition temperature, the degradation of PGA is temperature sensitive (Figure 8) and can be modeled with the Arrhenius equation [18]. 
The fracture toughness of the PGA plastics is also temperature sensitive and will change abruptly around the glass transition temperature of the material. The impact strength of PGA is highest near $60^{\circ} \mathrm{C}\left(140^{\circ} \mathrm{F}\right)$ and can change by a factor of eight between the glass transition temperature and higher or lower temperatures, as shown in Figure 9 [19]. This reduced impact strength at different temperatures can lead to chips, cracks, or other mechanical failures in hydraulic fracturing tools based on PGA plastic [17].

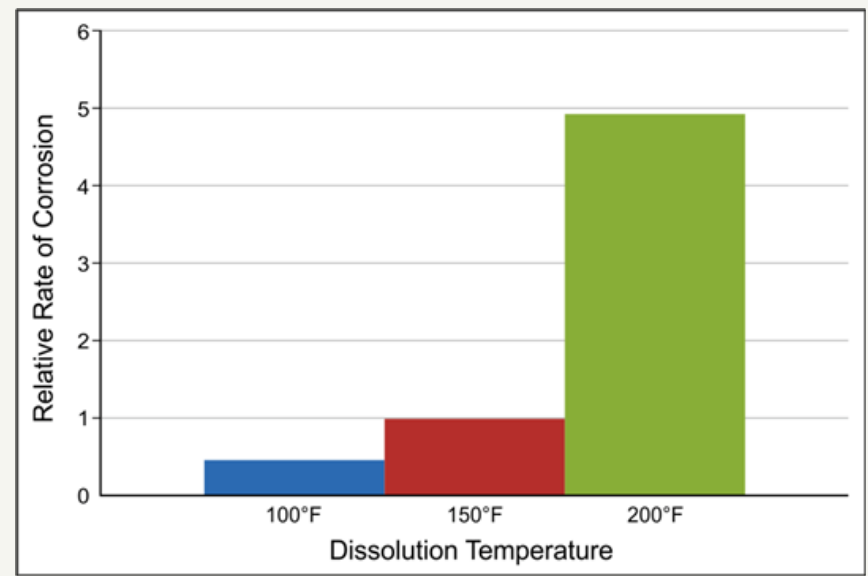

Figure 5: Relative rates of corrosion of dissolvable metal.

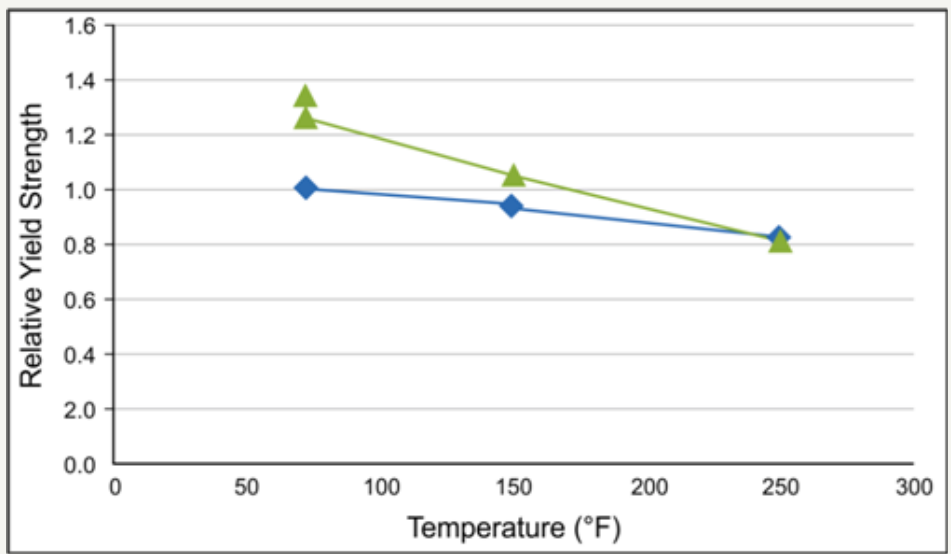

Figure 6: Relative yield strength for two different dissolvable metal alloys.

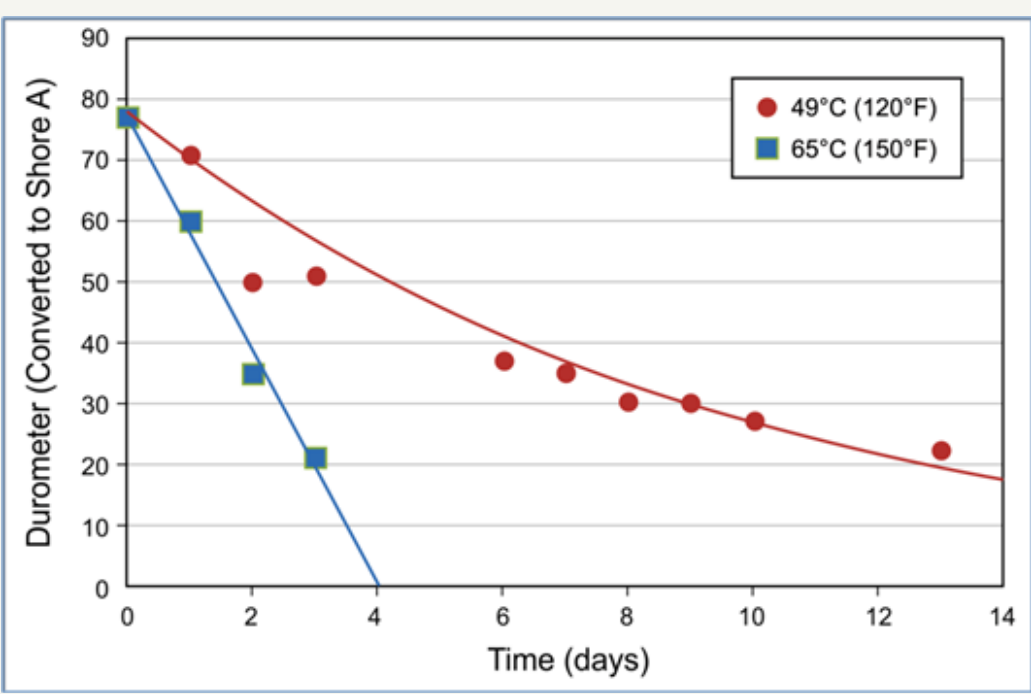

Figure 7: Degradation of low-temperature elements at 65 and $49^{\circ} \mathrm{C}\left(150\right.$ and $\left.120{ }^{\circ} \mathrm{F}\right)$. 


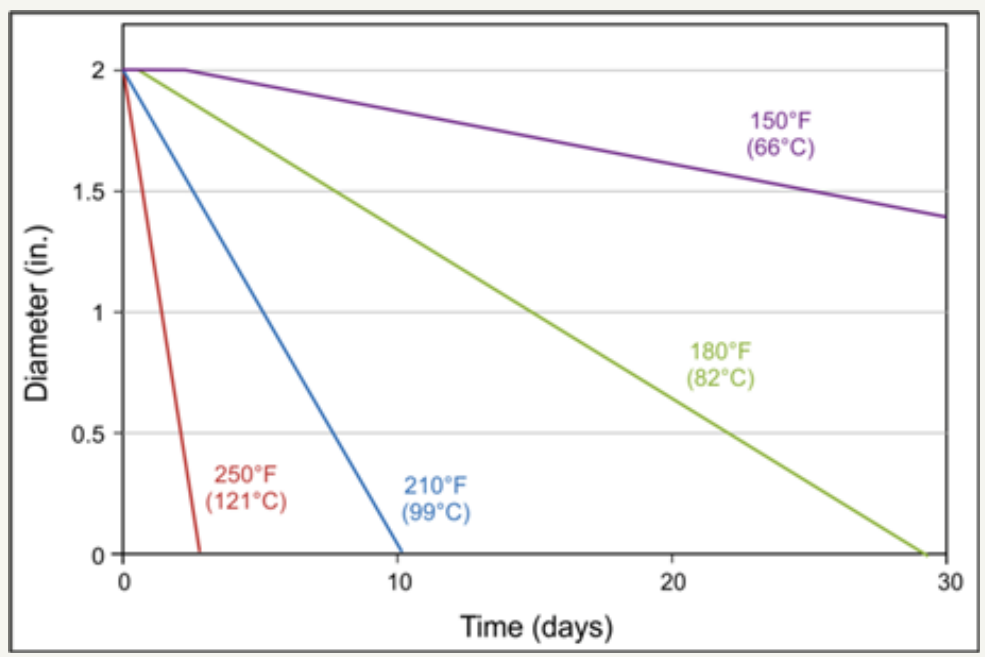

Figure 8: Temperature sensitivity of the degradation of PGA plastic [18].

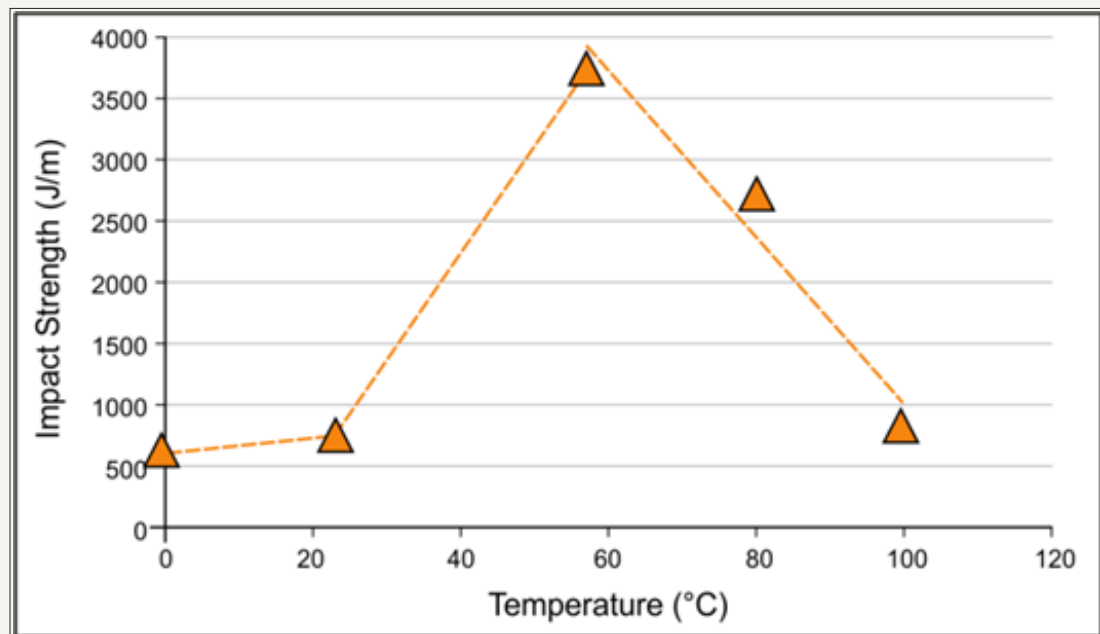

Figure 9: Temperature sensitivity of the impact strength of PGA plastic [18].

Discussion and Conclusion
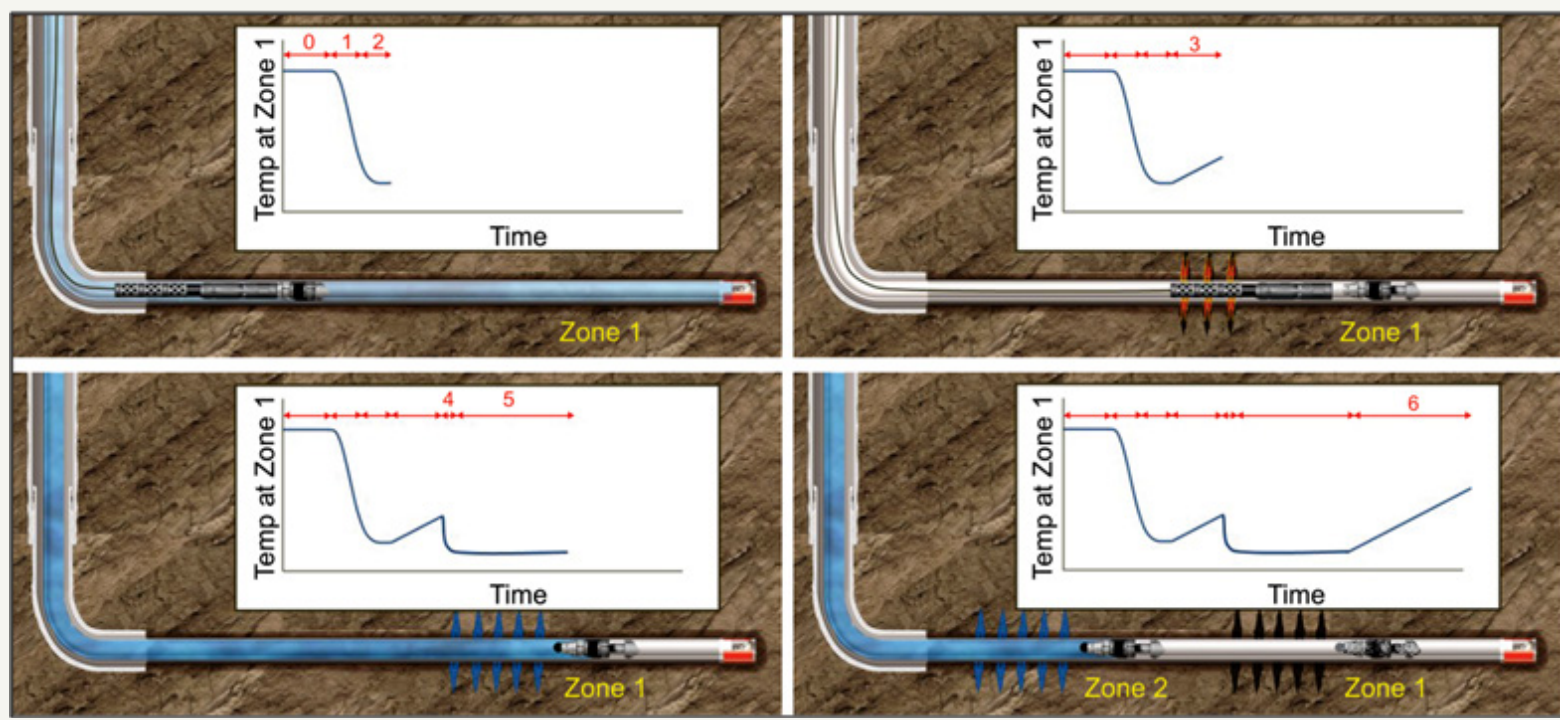

Figure 10: Down hole temperature, such as at the frac plug for Zone 1, varies during the hydraulic fracturing process. 
The hydraulic fracturing process involves injecting stimulation fluids into the formation in order to create additional cracks in the formation to allow the oil or gas to flow. The injected fluids are initially at the ambient temperature of the surface and will cool the casing during production operations. After the fracturing of a given stage is completed, the casing will reheat as heat flows from the formation. The cooling of the wellbore creates a dynamic temperature profile around the hydraulic fracturing tool. Figure 10 schematically illustrates the operational sequence for hydraulic fracturing. The down hole temperature is plotted at a location corresponding to where the frac plug for Zone 1 is set. Initially, during Stage 0, the wellbore is at the static down hole temperature that corresponds to the static down hole temperature [20]. The wellbore is rapidly cooled during Stages 1 and 2 while the perforating guns, the frac plug, and its setting tool are pumped to location. The wellbore will warm once fluid is no longer injected into the wellbore. Thus, during Stage 3 , the temperature increases during the perforating, pulling the guns out of the hole, and rigging up for the fracturing operation. Once fracturing commences with the dropping of the frac ball in Stage 4, the down hole temperature dramatically drops and remains low during the fracturing operation of Stage 5. Once the fracturing of that zone is completed, Stage 6 , then the temperature will increase as the geothermal heat warms the zone. The temperature will increase, even if there is fracturing at other zones higher in the wellbore. The warming of the area around a dissolvable frac plug will increase its rate of dissolution.

Cool down of the wellbore simplifies using dissolvable materials for hydraulic fracturing. The frac plug is cooled during the times when the plug is sealing against the highest differential pressure. The frac plug should have high strength during the hydraulic fracturing operation, as represented by Stage 5 in Figure 10, and these are periods when the wellbore is significantly cooler than the formation temperature. The frac plug is warmed during the times when the material should dissolve such as during Stage 6. It is desirable for the dissolvable frac plug to have minimal dissolution until after the fracturing is completed. It is during the reheating of the wellbore in Stage 6 when the dissolvable materials will degrade in the wellbore fluid. If an operational complication results in an extended delay between installation and stimulation (an extended warmback in Stage 3), then pumping a small amount of additional fluid before dropping the frac ball has been shown to rapidly cool the casing to near surface temperature.

The frac plug should be pressure tested at conditions similar to the actual dynamic downhole temperature, which will be cooler than static formation temperature and warmer than surface temperature. Many of the previous generation of composite frac plugs were tested at the static formation temperature (Guoynes et al. 1998) (Stegent and Howell 2009), which resulted in an overdesign of the tool. Equivalently, pressure testing the frac plug at room temperature fails to expose the frac plug to a representative down hole condition. The dynamic tool temperature can be accurately simulated with a thermal and flow simulator, such as that performed for Figure 1. This simulation allows for modeling the thermal conductivity of the reservoir, the cooling effect from previously stimulated zones, and geometry and distance from the actual wellbore. However, pressure testing the frac plug at $150{ }^{\circ} \mathrm{F}$ seems to provide a rough guide for hydraulic fracturing in many shale formations.

\section{References}

1. Xu L, He K, Rane J, Yin X, Neeves K (2015) Spontaneously imbibed fluids for increasing contact area between hydraulic fracturing fluids and formation matrix in liquids-rich shale plays. Society of Petroleum Engineers.

2. Eberhard M, Meijs R, Johnson J (2003) Application of flow-thru composite frac plugs in tight-gas sand completions. Society of Petroleum Engineers.

3. Fripp M, Walton Z (2016) Degradable metal for use in a fully dissolvable frac plug. Offshore Technology Conference.

4. Walton Z, Porter J, Fripp M, Vargus G (2017) Cost and Value of a Dissolvable Frac Plug. Society of Petroleum Engineers

5. Gil IR, Roegiers JC, Moos D (2006) Wellbore cooling as a means to permanently increase fracture gradient. Society of Petroleum Engineers.

6. Maury V, Guenot A (1995) Practical advantages of mud cooling systems for drilling. Society of Petroleum Engineers.

7. Johnson DO, Sierra JR, Gualtieri D, Kaura JD (2006) DTS transient analysis: a new tool to assess well flow dynamics. Society of Petroleum Engineers.

8. Gorgi B, Medina E, Gleaves J, Acuna J, Perera L, et al. (2014) Wellbore monitoring in carbonate reservoirs: value of DTS in acid stimulation through coiled tubing. Society of Petroleum Engineers.

9. Reyes RP, Yeager VJ, Glasbergen G, Parrish JL, Tucker R (2011) DTS sensing: an emerging technology offers fluid placement for acid. Society of Petroleum Engineers

10. Sierra JR, Kaura JD, Gualtieri D, Glasbergen G, Sarkar D, et al. (2008) DTS monitoring of hydraulic fracturing: experiences and lessons learned. Society of Petroleum Engineers.

11. Holley EH, Molenaar MM, Fidan E, Banack B (2012) Interpreting uncemented multistage hydraulic-fracturing completion effectiveness by use of fiber-optic DTS injection data. Society of Petroleum Engineers.

12. Wheaton B, Haustveit K, Deeg W, Miskimins J, Barree R (2016) A case study of completion effectiveness in the eagle ford shale using DAS/ DTS observations and hydraulic fracture modeling. Society of Petroleum Engineers.

13. Tabatabaei M, Zhu D (2011) Fracture stimulation diagnostics in horizontal wells using DTS data. Society of Petroleum Engineers.

14. Halliburton (2013) WELLCAT Manual. Halliburton, Houston, Texas, USA.

15. Walton Z, Fripp M, Merron M (2016) Dissolvable metal vs. dissolvable plastic in downhole hydraulic fracturing applications. Offshore Technology Conference.

16. Fripp M, Walton Z, Norman T (2017) Fully dissolvable fracturing plug for low-temperature wellbores. Society of Petroleum Engineers.

17. Lyu S, Untereker D (2009) Degradability of polymers for implantable biomedical devices. Int J Mol Sci 10(9): 4033-4065.

18. Okura M, Takahashi S, Kobayashi T, Saijo H, Takahashi T (2015) Improvement of impact strength of polyglycolic acid for self-degradable tools for low-temperature wells. Society of Petroleum Engineers. 
19. Guoynes JC, Toothman RL, Berscheidt K, Malimasura PR (1998) New composite fracturing plug improves efficiency in coalbed methane completions. Society of Petroleum Engineers.
20. Stegent NA, Howell M (2009) Continuous multistage fracture stimulation completion process in a cemented wellbore. Society of Petroleum Engineers. (c) Creative Commons Attribution 4.0

For possible submissions Click Here

\begin{tabular}{|c|} 
Progress in Petrochemical Science \\
Benefits of Publishing with us \\
- High-level peer review and editorial services \\
- Freely accessible online immediately upon publication \\
- Authors retain the copyright to their work \\
- Licensing it under a Creative Commons license \\
- Visibility through different online platforms
\end{tabular}

